
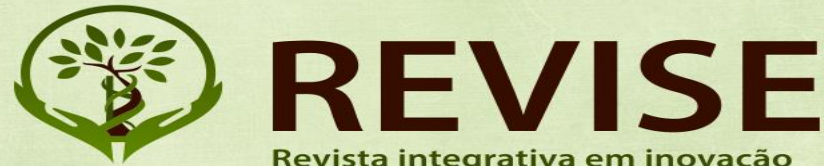

Revista integrativa em inovação tecnológica nas ciências da saúde

ISSN: $2179-6572$

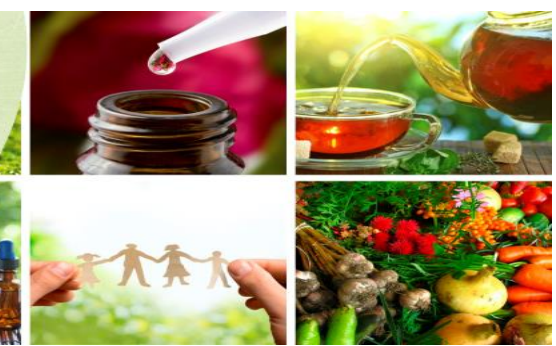

\title{
GESTAÇÃO, PARTO E PUERPÉRIO NA PERSPECTIVA DAS GESTANTES DE UMA UNIDADE BÁSICA DE SAÚDE
}

\section{GESTATION, CHILDBIRTH AND PUERPERIUM IN THE PERSPECTIVE OF PREGNANT WOMEN FROM A BASIC HEALTH UNIT}

\author{
Larissa da Silva Vieira \\ Jeniffer de Araújo Abreu \\ Karen Kessy Souto Paulo \\ Lavínia da Silva Menezes \\ Luiza Bastos Martins \\ Maria Luiza Caires Comper \\ UFSB
}

\section{RESUMO}

A gravidez, o parto e o puerpério representam um ciclo de vida caracterizado por profundas mudanças e sucessivas adaptações biopsicossociais. As ações de educação em saúde constituem uma estratégia do cuidado e devem ser orientadas por uma escuta sensível, empática, de acolhimento e valorização das especificidades de cada mulher. O objetivo é relatar a experiência de uma prática pedagógica, do tipo roda de conversa, realizada com um grupo de gestantes em uma Unidade Básica de Saúde. O relato descreve os achados e os aspectos vivenciados por estudantes do Bacharelado Interdisciplinar em Saúde durante a realização de uma atividade pedagógica do componente curricular de Semiologia dos problemas de Saúde da Gestação, Parto e Puerpério. Uma roda de conversa foi conduzida com um grupo de gestantes que realizavam consultas em uma Unidade Básica de Saúde, localizada na cidade de Itabuna/BA. A coleta de dados foi feita por meio de técnicas de observação direta e participante. A experiência possibilitou reconhecer que as mulheres gestantes possuem dúvidas, medos e anseios quanto à gravidez, parto e puerpério. ${ }^{1}$ As ações

\footnotetext{
${ }^{1} \mathrm{O}$ estudo não recebeu financiamento.
} 

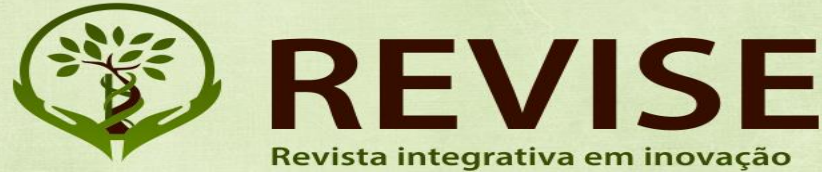

Revista integrativa em inovação
tecnológica nas ciências da saúde ISSN: $2179-6572$

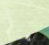

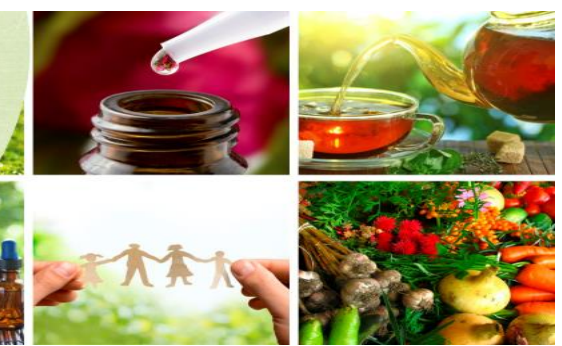

educativas oportunizaram a troca de saberes entre gestantes e estudantes que os dotou de mais conhecimento, autonomia e capacidade crítica.

Palavras-Chave: Atenção Primária à Saúde. Educação em Saúde. Saúde da Mulher.

\begin{abstract}
Pregnancy, childbirth and the puerperium represent a life cycle characterized by profound changes and successive biopsychosocial adaptations. Health education actions are a care strategy and should be guided by sensitive and empathetic listening, welcoming and valuing the specificities of each woman. The aim is to report the experience of a pedagogical practice with a circle conversation carried out with a group of pregnant women from a Basic Health Unit. The report describes the findings and aspects experienced by the students of the Interdisciplinary Bachelor in Health when they carrying out a pedagogical activity on the Semiology of the problems of Gestational Health, Childbirth and Puerperium curriculum component. A conversation circle was held with a group of pregnant women who performed consultations at a Basic Health Unit, located in the city of Itabuna/BA. Data collection was performed using direct and participant observation techniques. The experience made it possible to recognize that pregnant women have doubts, fears and anxieties in relation to pregnancy, childbirth and the puerperium. Educational actions enabled the exchange of knowledge between pregnant women and students, which provided them with more knowledge, autonomy and critical capacity.
\end{abstract}

Keywords: Primary Health Care. Health Education. Maternal Health.

\title{
INTRODUÇÃO
}

A representação da mulher e seus significados tem sofrido inúmeras mudanças ao longo da história. Uma das mais importantes, reside na autonomia de decisão sobre a maternidade, a idade para engravidar, o número de filhos, e a escolha do método anticoncepcional mais adequado. O Planejamento Familiar (PF), realizado gratuitamente e universalmente pelo Sistema Único de Saúde, fomenta ações que auxiliam as pessoas que pretendem ter filhos e também aquelas que preferem adiar 


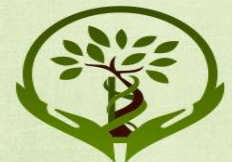

ISSN: $2179-6572$

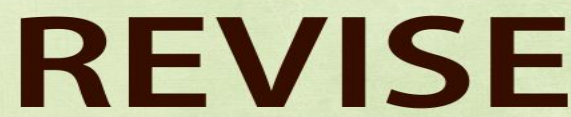

Revista integrativa em inovação tecnológica nas ciências da saúde

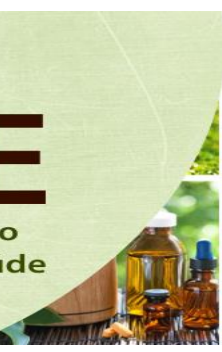

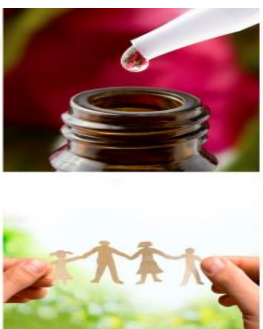

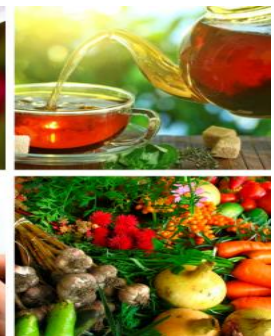

o aumento da família (BRASIL, 1996). Isso porque, o PF democratiza o acesso aos métodos contraceptivos e conceptivos por meio da saúde pública, ao mesmo tempo que auxilia as gestantes a enfrentarem esse período de tantas mudanças de forma que seja benéfico para a mesma (ANDRADE; SILVA, 2009).

Diante da concepção, a mulher é acompanhada pela equipe de saúde durante a gestação, o parto e puerpério por meio da assistência pré-natal. O pré-natal deve ser iniciado a partir do momento que a gravidez for identificada, sendo preferencial que seu início se dê ainda no primeiro trimestre da gestação. O objetivo é atuar precocemente na prevenção e/ou detecção de patologias maternas e/ou fetais que possam gerar complicações para o desenvolvimento fetal ou para a saúde materna (BRASIL, 2012). Além disso, o acolhimento dessa mulher desde o início da gestação favorece a manutenção de seu bem-estar físico e emocional (JARDIM et al., 2019).

Esse cuidado durante o pré-natal é fundamental para a mulher que se encontra no ciclo gravídico-puerperal, uma vez que se trata de um processo fisiológico que compreende profundas mudanças decorrentes das alterações biológicas, somáticas e psicológicas (MANN et al., 2010; PICCININI et al., 2008). Além disso, muitas vezes a mulher ainda tem que lidar com as mudanças que ocorrem no campo ocupacional e social, tendo que se adaptar a essa nova configuração da vida (SILVEIRA; FERREIRA, 2011). Por isso, é importante priorizar estratégias que contribuam para o empoderamento, enfrentamento e adaptação às mudanças, com ações voltadas para a superação de medos, dúvidas e dificuldades (TOSTES; SEIDL, 2016).

A educação em saúde é uma das estratégias utilizadas pela Atenção Básica para aumentar a autonomia das mulheres no seu cuidado durante o ciclo gravídico-puerperal (SOUZA et al., 2011). Para que esse resultado seja alcançado com efetividade é indispensável que os profissionais de saúde compreendam a realidade social, cultural e econômica de cada gestante, de maneira que a construção do conhecimento esteja apropriada e qualificada às demandas da população (ANDRADE et al., 2013). As ações educativas não devem ser somente informativas. Ao contrário, devem oportunizar espaços de reflexão que tornem as mulheres agentes de mudança de seus próprios processos (PIO; OLIVEIRA, 2014).

Diante do exposto, objetiva-se relatar a experiência de uma prática pedagógica, do tipo roda de conversa, realizada com um grupo de gestantes em uma Unidade Básica de Saúde. O relato 


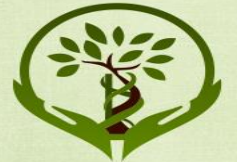

Revista integrativa em inovação tecnológica nas ciências da saúde

ISSN: 2179-6572
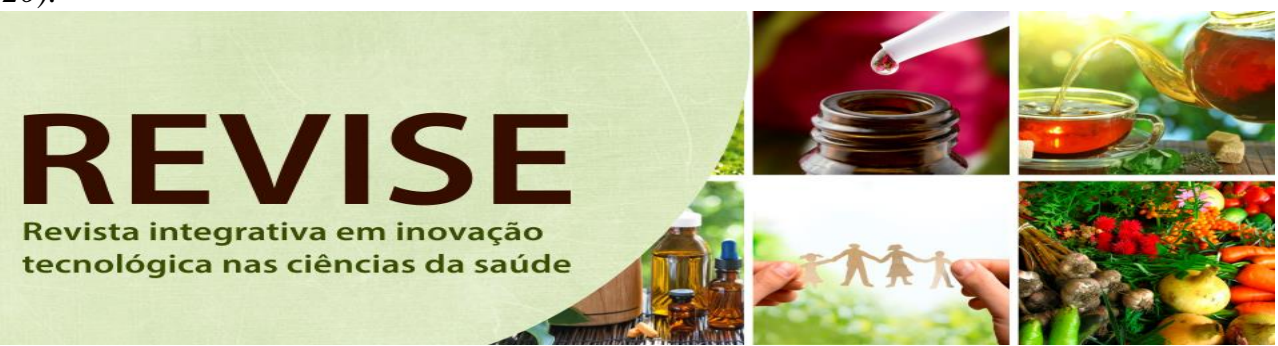

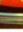
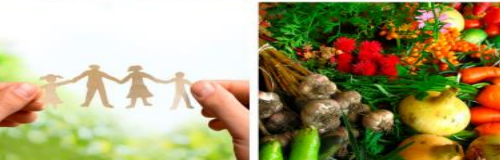

descreve os achados e os aspectos vivenciados por estudantes do Bacharelado Interdisciplinar em Saúde durante a realização de uma atividade pedagógica do componente curricular de Semiologia dos Problemas de Saúde da Gestação, Parto e Puerpério.

\section{MÉTODOS}

\section{Desenho de Estudo}

Trata-se de um estudo descritivo, do tipo relato da experiência, que descreve os aspectos vivenciados por um grupo de estudantes do Bacharelado Interdisciplinar em Saúde (BIS) durante o Componente de Propedêutica Clínica dos Problemas de Saúde da Gestação, Parto e Puerpério. Este componente pertence ao Bloco Temático "Integração de Competências Clínicas" e compreende conhecimentos e habilidades necessárias para obtenção, compreensão e análise de dados de importância clínica.

Em termos mais específicos, o componente curricular de Propedêutica Clínica dos Problemas de Saúde da Gestação, Parto e Puerpério se propõe a apresentar e discutir as inter-relações entre processos morfofuncionais e fisiopatológicos articulados às especificidades de cada trimestre gestacional, bem como a sua correlação com os principais problemas de saúde neste ciclo de vida; e, aprofundar competências e habilidades básicas para a produção de dados significativos aplicados à gestação, parto e puerpério.

A estratégia pedagógica utilizada foi de aprendizagem híbrida com estrutura de "aula invertida". Os estudantes tiveram acesso aos conteúdos de cada sessão antecipadamente, por meio do Plano de Atividades (PLAT) detalhado, contendo horários, tópicos, objetivos, tarefas, metodologias, instruções e referências. O PLAT e outros conteúdos foram disponibilizados pela plataforma Moodle. Os estudantes foram organizados em Equipes de Aprendizagem Ativa (EAA) formadas por três a cinco participantes.

Como estratégia pedagógica foi proposta a realização de tarefas extraclasse que incluía a realização de práticas educativas com gestantes acompanhadas em alguma Unidade Básica de Saúde (UBS) do município. Essas tarefas incluíram a realização de práticas de acolhimento, ações 

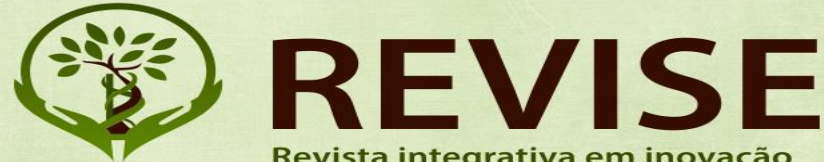

Revista integrativa em inovação tecnológica nas ciências da saúde

ISSN: 2179-6572

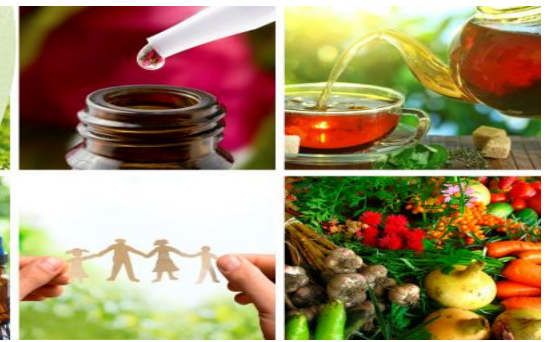

educativas, vivências e observações, entrevistas e outros. Os estudantes foram orientados a registrar imagens, observações, relatos e percepções em um relatório a ser disponibilizado ao final do CC.

Cada EAA escolheu, por conveniência, a UBS do município onde seriam realizadas as tarefas. Para este relato, considerou-se a Unidade Básica de Saúde José Maria Magalhães Neto, pertencente ao módulo III, no município de Itabuna - BA. Esta UBS foi selecionada em razão de suas características de atuação, uma vez que está localizada no centro da cidade, possui 02 equipes de saúde da família, abrange cerca de 13 microáreas de saúde do município e atende a demanda dos principais bairros do município de Itabuna, incluindo o Centro, Pontalzinho, Castália, Alto Mirante, Alto Maron e Parque São João.

O município de Itabuna está localizado no Sul da Bahia e possui uma população estimada no ano de 2018 de cerca de 212.740 mil habitantes, com densidade demográfica de 473,50 hab/km² (IBGE, 2018). A Região de Saúde de Itabuna atende 22 municípios circunvizinhos, com um total de 532.999 habitantes. O município também é referência para média e alta complexidade de saúde da Microrregião Itabuna-Ilhéus e Macrorregião do Sul da Bahia.

\section{Descrição da Intervenção de Educação em Saúde}

A intervenção de educação em saúde foi conduzida em três etapas, como pode ser observado na Figura 1. Inicialmente, realizou-se o primeiro contato com o responsável administrativo da UBS para saber se haveria a possibilidade de realização da intervenção. Após a confirmação de viabilidade e aceite, os estudantes realizaram uma entrevista com as enfermeiras responsáveis pelo pré-natal da UBS com o objetivo de identificar o perfil, as principais demandas, os anseios e dúvidas das gestantes que estavam sendo acompanhadas. Essas informações foram utilizadas para planejamento do convite de divulgação e da intervenção em si. O convite de divulgação da intervenção foi enviado para as gestantes por meio de um grupo de conversa que elas possuem no aplicativo de troca de mensagens instantâneas WhatsApp.

A intervenção do tipo roda de conversa ocorreu em um único dia, no refeitório da UBS no intervalo da realização do pré-natal. Ferramentas da educação popular em saúde foram utilizadas para proporcionar uma comunicação culturalmente competente e horizontal, capaz de produzir e ressignificar saberes, fomentar a autonomia e o empoderamento das participantes (BRASIL, 2014). 

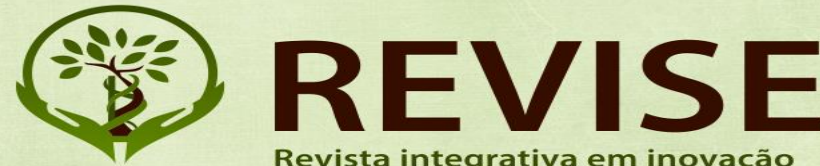

Revista integrativa em inovação tecnológica nas ciências da saúde

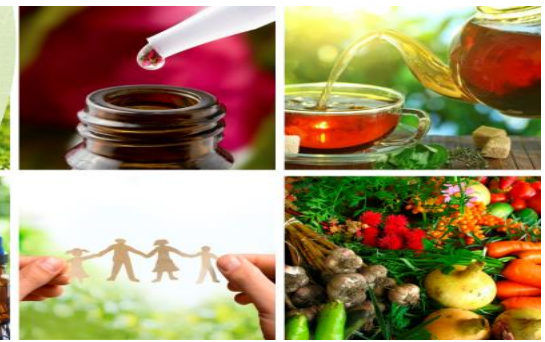

Por isso, todas as participantes puderam expressar suas opiniões, concepções, impressões e conceitos sobre o período gestacional e puerperal de forma livre. Um roteiro com 12 (doze) perguntas relacionadas à gestação, parto e puerpério foi utilizado como apoio para mediar as discussões. Ao final, os estudantes apresentaram um infográfico, no formato de pôster, com síntese de informações importantes a serem consideradas durante a gestação, parto e puerpério.

A coleta de dados foi feita por meio de técnicas de observação direta e participativa durante a roda de conversa. Registros fotográficos e anotações foram feitas, após serem autorizadas pelas participantes. Posteriormente, as informações obtidas foram transcritas e organizadas em um documento do software Microsoft Word (versão 2007).

O estudo em questão dispensa a aprovação do Comitê de Ética em Pesquisa (CEP) tendo em vista que se trata de um relato de experiência onde os participantes da pesquisa não foram identificados, de acordo com a Resolução n 466/12 e a Resolução n. 510/16 do Conselho Nacional de Saúde (CONSELHO NACIONAL DE SAÚDE, 2013, 2016).

Figura 1 - Etapas da realização de Diagnóstico e Planejamento da Intervenção de Educação em Saúde para Gestantes
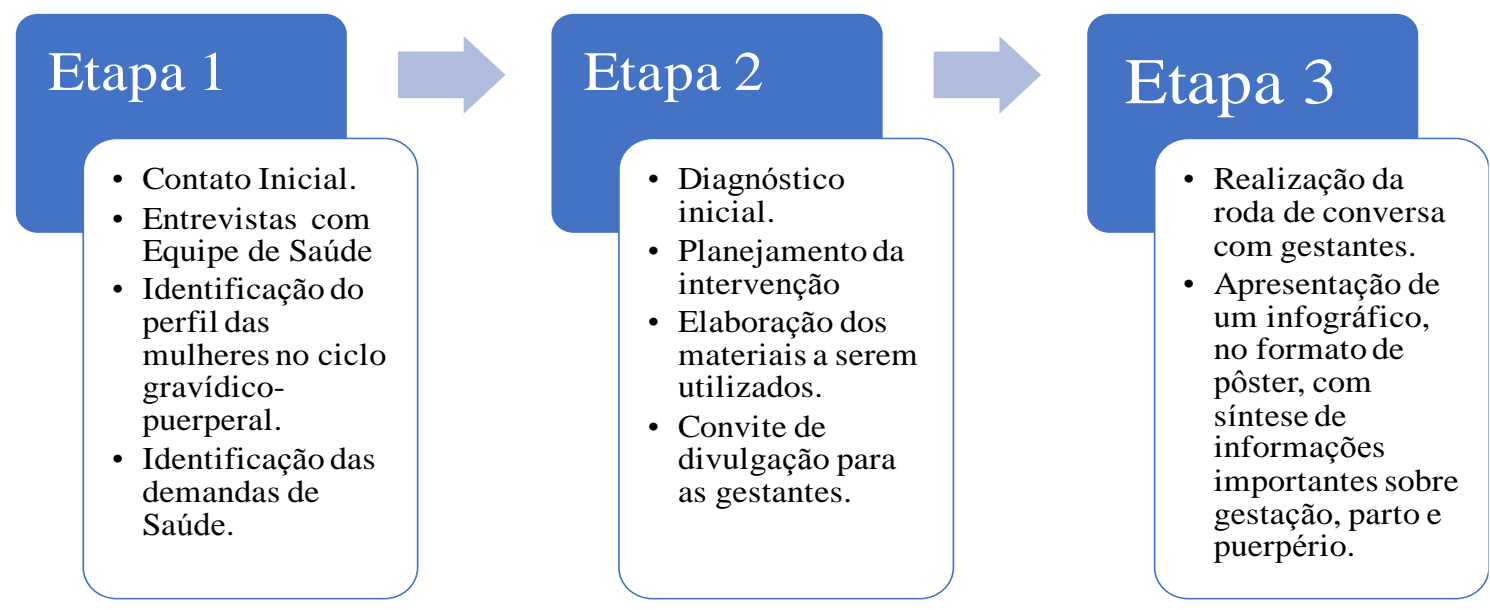

Fonte: Vieira et al. (2019) 

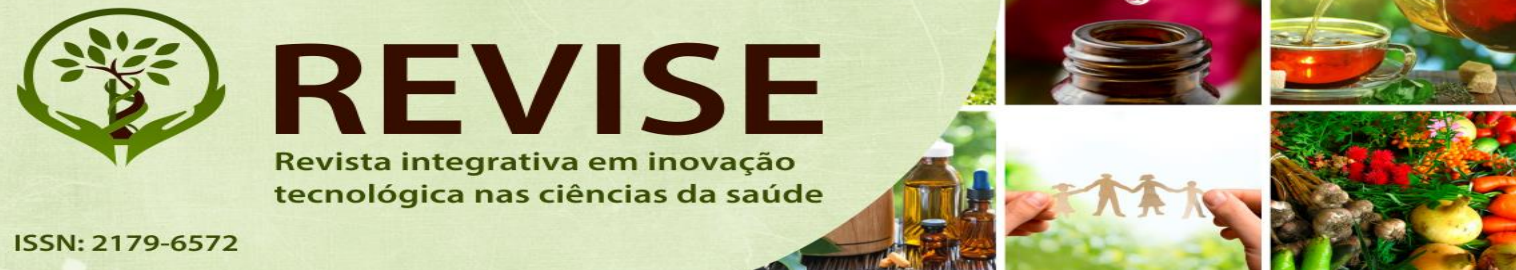

\section{RESULTADOS}

\section{Descrevendo os principais resultados da intervenção}

Participaram do estudo um grupo de 07 (sete) gestantes com idade igual ou superior a 18 anos. Todas possuíam o $2^{\circ}$ grau completo e, em sua maioria, residiam ou trabalhavam no comércio da área de abrangência da UBS mencionada. A maioria das participantes eram primigestas. Cinco delas relataram ter realizado o planejamento da gestação juntamente com o marido e/ou companheiro. As demais relataram que, apesar de não terem engravidado de modo planejado, estavam lidando bem com a notícia, assim como a família, demonstrando felicidade e afeto à gravidez. Apenas uma gestante mostrou-se receosa devido a diferença de idade da sua primeira gestação para a atual. Nenhuma gestante relatou praticar atividade física antes da gestação.

Os métodos contraceptivos que eram mais utilizados foram o anticoncepcional oral, injetável e a tabelinha. $\mathrm{O}$ uso da camisinha foi relatado por somente uma gestante. O principal motivo relatado para não utilizar esse método de barreira é o fato de possuírem apenas um parceiro sexual. Em relação ao sexo, praticamente todas as mulheres relataram que a gravidez não modificou o interesse e a frequência das relações sexuais. Somente uma relatou incômodo e ausência de desejo sexual. No entanto, algumas delas afirmaram que seus parceiros demonstram medo e/ou insegurança para isso.

A maior parte das gestantes perceberam alterações físicas e psicológicas que afetaram de alguma forma seus hábitos de vida. As principais foram: cansaço, náuseas, vômitos, oscilações de humor, alterações da pressão arterial, excesso de sede, presença de edema nos membros inferiores, sensibilidade e aumento nas mamas, escurecimento da pele, estrias, mudanças no fio do cabelo e aumento da quantidade capilar corporal. Houveram poucos relatos sobre intercorrências. As que houveram estavam relacionadas às mudanças na pressão arterial, infecção vaginal, sobrepeso e incompatibilidade sanguínea do feto x mãe. A ansiedade foi relatada pelas gestantes com frequência, principalmente em relação a expectativa do momento do parto.

Em relação à preferência do tipo de parto, as opiniões das gestantes ficaram divididas. No entanto, o parto normal parece ter uma aceitação melhor. As gestantes conhecem a importância da amamentação e demonstram desejo frequente em poder amamentar seus filhos do nascimento até quando necessário. Dentre as dúvidas e inseguranças relacionadas a esse tema, foram citados: o 

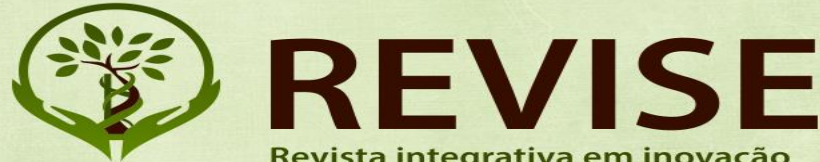

Revista integrativa em inovação tecnológica nas ciências da saúde

ISSN: $2179-6572$

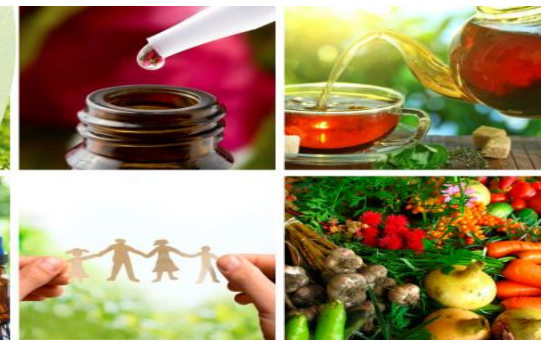

tamanho e formato do mamilo, modo de amamentar e o medo em não conseguir produzir leite. Todas as gestantes relataram poder contar com uma pessoa no pós-parto, seja o parceiro, a mãe ou a sogra. Entretanto, foi exposto a dificuldade em se locomover de uma cidade para outra em decorrência da necessidade dessa assistência. Além disso, foram partilhados angústias e anseios sobre esse período de mudança na vida da mulher.

\section{Descrevendo os principais resultados do processo formativo}

O percurso acadêmico construído pelo componente curricular Semiologia dos Problemas de Saúde na Gestação, Parto e Puerpério proporcionou uma ampla visão para integração de conhecimentos teóricos e práticos que culminaram no planejamento e realização da intervenção com as gestantes atendidas em uma UBS do município de Itabuna, Bahia. A compreensão das alterações morfofuncionais e psicossociais existentes no período gravídico-puerperal contribuiu para: (i) a coleta de informações durante as entrevistas com as gestantes e profissionais de saúde, (ii) análise das demandas e do perfil das gestantes, e (iii) condução da educação em saúde.

A atividade pedagógica do CC, ao integrar ensino-serviço, proporcionou uma aproximação dos estudantes ao contexto real de uma UBS e aos princípios do SUS. Planejar e conduzir uma intervenção de educação em saúde possibilitou uma experiência de gestão em saúde e de desenvolvimento de habilidades clínicas para produção de dados por meio da ética, escuta e acolhimento. A roda de conversa mobilizou conhecimentos e desenvolvimento da autonomia, do pensamento crítico-reflexivo e do discurso dos estudantes.

Ademais, a roda de conversa permitiu que o público-alvo (gestantes) contasse suas experiências de acordo com o que estavam vivendo naquele momento, com suas próprias percepções. Em alguns momentos estas acabavam por se tornar percepções coletivas, uma vez que as gestantes se identificavam entre si e viam, em outras histórias, similaridades com o seu processo de gestação. Com isso, adquirimos habilidades e competências referentes a escuta e mediação necessárias para conduzir a roda de conversa, além de nos permitir compreender a realidade ao qual uma gestação está ligada, levando-se em consideração que cada gestante é única, e que para uma mesma pessoa cada gestação também possui suas particularidades. 


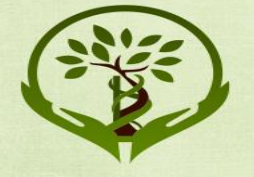

ISSN: $2179-6572$

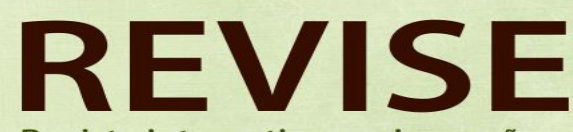

Revista integrativa em inovação tecnológica nas ciências da saúde
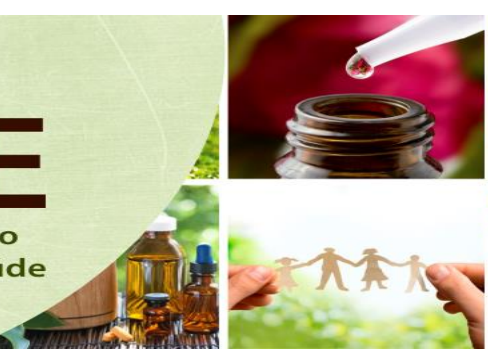

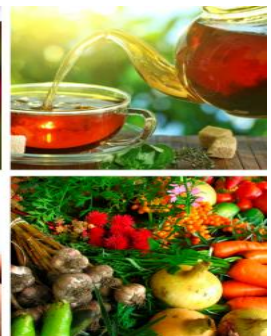

Cabe destacar que a experiência também resultou na observação de limitações e obstáculos que podem dificultar a realização de práticas educativas em saúde. A principal limitação é a frequência e o curto espaço de tempo disponível para a prática educativa. Muitas gestantes só são liberadas do trabalho nos dias e horários agendados para atendimento do pré-natal, o que limita ações mais duradouras. A outra limitação encontrada foi a resistência de alguns profissionais de saúde no acolhimento de estudantes, visto que necessitam explicar a rotina do serviço de saúde e adequar os atendimentos das unidades para às atividades destes. Nesse sentido, a agenda pré-estabelecida no serviço de saúde e o alto fluxo de pacientes em dias específicos foram fatores limitantes encontrados.

\section{DISCUSSÃO}

A experiência alcançada durante a realização de uma atividade pedagógica que inclui o planejamento e a execução de uma intervenção de educação em saúde com gestantes em uma roda de conversa foi positiva tanto para o processo formativo dos estudantes quanto para as mulheres gestantes que puderam expressar suas dificuldades, mudanças e anseios. É possível reconhecer que a roda de conversa se tornou um espaço simbólico e campo para promoção da educação em saúde, em que foi possível esclarecer dúvidas sobre os diferentes tipos de parto, destacando as vantagens do parto normal como principal escolha das gestantes; sobre a importância da amamentação e o uso de contraceptivos de barreira e anticoncepcional oral e injetável como principais métodos contraceptivos a serem adotados no período do puerpério.

Esse resultado corrobora com Freire (2011) o qual defende que a educação libertadora está vinculada à conscientização das pessoas, uma vez que para se alcançar o êxito em determinada ação, deve-se haver a participação daqueles que estão sendo educados. Dessa maneira, torna-se importante a inserção de rodas de conversa como estratégia de práticas educativas em saúde. Isso porque a educação contextualizada demarca a imersão dos indivíduos engajados no ato de transformar e conhecer a realidade de cada um, condicionando os participantes a reconstruir a sua existência através do conhecimento obtido (SAMPAIO et al., 2014).

Cabe destacar que as falas das gestantes construíram pontes, tanto para o fluxo de troca de experiência quanto para a interlocução do processo formativo, possibilitando um diagnóstico dos 


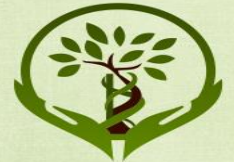

Revista integrativa em inovação tecnológica nas ciências da saúde
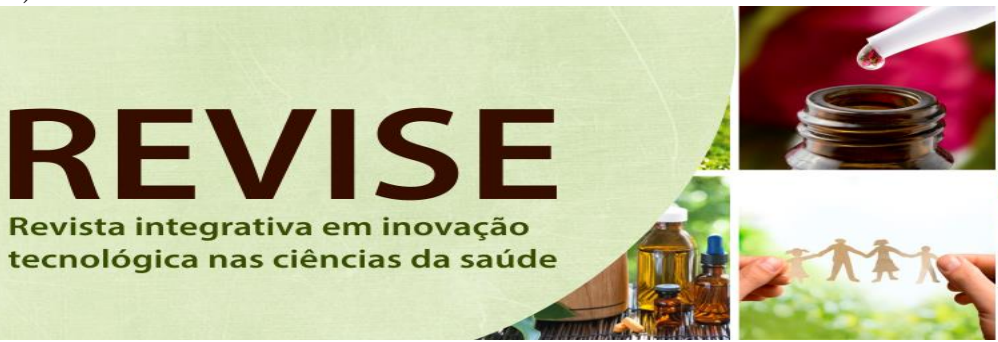

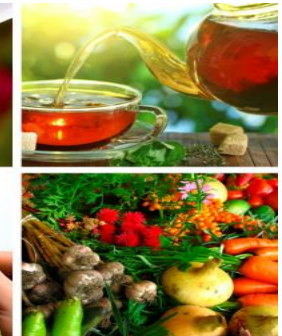

principais anseios, dúvidas e dificuldades vivenciadas pelas experiências das gestantes. Este achado também foi observado por Souza (2014) ao obter 95\% de satisfação das gestantes que participaram de rodas de conversas em saúde como metodologia participativa no acompanhamento do pré-natal.

Em relação aos achados obtidos durante a intervenção, destaca-se que uma das principais dúvidas está relacionada ao ato sexual e as mudanças decorrentes da gestação. De fato, alguns estudos relatam que a gestação pode resultar em disfunção sexual, cujas principais causas estão relacionadas à diminuição da libido, sangramentos vaginais, dor no baixo ventre e o medo de que o bebê fosse prejudicado pela relação sexual são situações que, com frequência, interferem na vida sexual do casal (ARAÚJO et al., 2012; PRADO et al., 2013). Apesar desses achados, vale ressaltar que a atividade sexual é algo natural, biológico, fisiológico e um dos fatores que mais promovem prazer ao indivíduo. Portanto, não se deve inibir a sexualidade no período da gravidez. Ao contrário, a mulher gestante deve ter uma vida sexual ativa como alternativa para manutenção do bem-estar físico, emocional e psicológico da mulher e do casal (COUTINHO et al., 2014).

Do mesmo modo, recomenda-se que a camisinha deve ser utilizada para prevenir a transmissão de Infecções Sexualmente Transmissíveis (IST's), especialmente durante a gravidez. Essas infecções podem ser transmitidas por outros meios, além de relações extraconjugais. Além disso, durante a gestação, o contágio das IST's pode prejudicar a saúde da gestante e do bebê, trazendo complicações para o desenvolvimento da criança, bem como a transmissão da doença para o bebê durante o parto. Os fatores biológicos e sociais podem fazer com que as mulheres fiquem mais vulneráveis a doenças como HIV/Aids e as IST's quando comparadas aos homens (BRUM, 2013).

Em relação às mudanças morfofuncionais e psicossociais, a maior parte das gestantes relataram os seguintes sintomas: cansaço, náuseas, vômitos, oscilações de humor, alterações da pressão arterial, excesso de sede, presença de edema nos membros inferiores, sensibilidade e aumento nas mamas, escurecimento da pele, estrias, mudanças no fio do cabelo e aumento da quantidade capilar corporal. Tais sintomas são próprios da gestação, enquanto um período de intensas transformações que envolvem variados sistemas e aparelhos do corpo humano (SILVA et al., 2015). Dentre essas transformações, destacam-se o aumento das mamas, do abdômen e do peso corporal no segundo e terceiro semestres da gestação (COSTA et al., 2010), além de fadiga, náuseas, amenorreia, dor nas mamas, sangramento gengival, dor lombar, edema e câimbras (QUEROZ, 2012). 


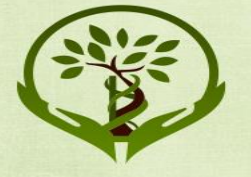

ISSN: $2179-6572$

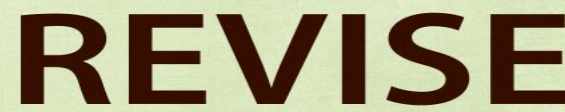

Revista integrativa em inovação tecnológica nas ciências da saúde

\section{西}

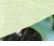

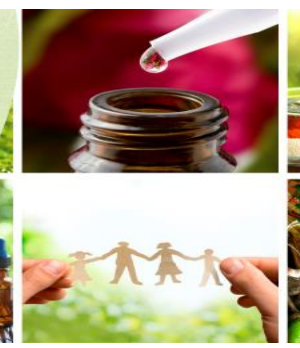

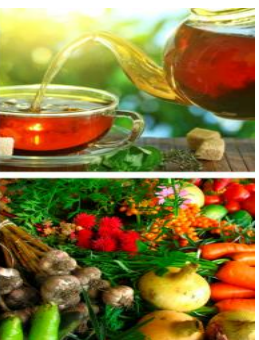

A expectativa pelo momento do parto se mostrou como principal causa de ansiedade. Muitas mulheres quiseram conhecer as características de cada tipo de parto, incluindo as situações de recomendação, os riscos e os benefícios. Salientamos a importância de resguardar o direito à gestante em escolher o tipo de parto, recomendando o parto normal como ideal, pois além de uma recuperação menos dolorosa ele possibilita a mulher adotar uma postura ativa, movimentar o seu corpo, exercer o protagonismo do momento, cabendo aos profissionais de saúde auxiliar no necessário (VELHO et al., 2014). Realizar orientação sobre a amamentação e seus benefícios também é importante, considerando que o contexto sociocultural e familiar influencia muito na tomada de decisão de introdução de novos alimentos e desmame (ANDRADE et al., 2015).

Um outro achado relevante foi a ausência de atividade física durante a gestação por todas as participantes da intervenção. A prática de atividade física é recomendada para a manutenção e promoção da saúde da gestante, reduz consideravelmente fatores de risco gestacional e puerperal, quando realizada mediante orientação profissional e adequada às suas necessidades. Dessa forma, por ser um período único da vida de uma mulher, torna-se pertinente adotar comportamentos de estilo de vida saudáveis, incluindo a atividade física, a qual tem sido proposta como uma medida preventiva ou terapêutica para reduzir as complicações da gravidez e otimizar a saúde materno-fetal (MOTTOLA et al., 2019).

Diante do exposto, observa-se que a roda de conversa realizada explorou um campo duplo de aprendizado, visto que houve uma troca de conhecimentos e saberes, oportunizando uma experiência social e acadêmica que dificilmente seria possível no âmbito do atendimento técnico de saúde. Ademais, a intervenção possibilitou o reconhecimento da participação cooperativa profissionalpaciente nos processos de decisão e a associação da teoria aprendida em sala de aula, quanto às alterações morfofuncionais, fisiopatológicas e emocionais no período gestacional, à prática vivida na Unidade Básica de Saúde.

Além disso, é necessário salientar que a estratégia utilizada não só permite que a público-alvo aprenda com a experiência de outrem, mas também que os estudantes participantes desenvolvam e pratiquem a habilidade de escuta. $\mathrm{O}$ aprendizado é uma construção mútua, dessa forma, atestamos a importância de atividades que promovam a participação de profissionais, estudantes e comunidade, contribuindo assim para a transformação de mais mulheres no contexto social e da saúde. 

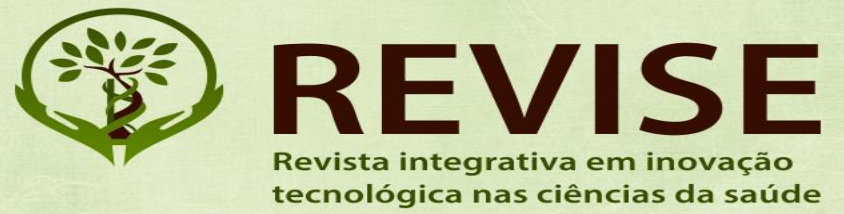

ISSN: $2179-6572$ tecnológica nas ciências da saúde
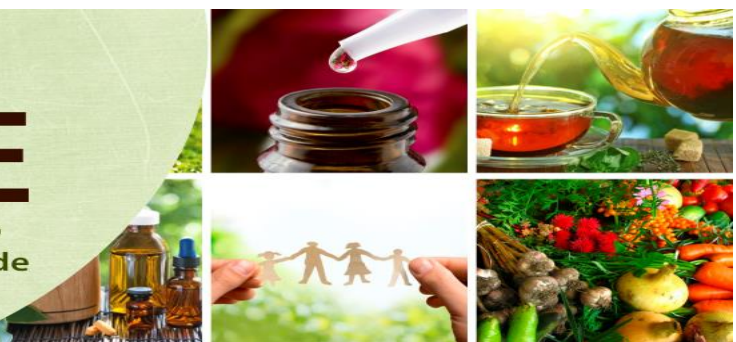

\section{CONSIDERAÇÕES FINAIS}

Para o ensino, a atividade pedagógica conduzida pelo CC de Semiologia dos Problemas de Saúde na Gestação, Parto e Puerpério cumpriu o objetivo de permitir o desenvolvimento de conhecimentos e habilidades importantes para a formação dos estudantes. A intervenção realizada por meio da roda de conversa com um grupo de gestantes demonstrou ser um momento de compartilhamento e apoio mútuo. Vivenciamos em uma situação real de UBS, os conhecimentos apreendidos nas aulas, percebendo a relevância do diálogo e acolhimento no cuidado integral à mulher.

Para o serviço, a realização de uma intervenção de educação em saúde mostrou a importância de ampliar o conhecimento das mulheres gestantes para o cuidado consigo mesma. Os resultados observados nos discursos podem contribuir para o planejamento de ações de saúde a serem realizadas na UBS. Por essa razão, é importante que a equipe multiprofissional da UBS promova atividades educativas e integrativas com as gestantes e puérperas e suas redes de apoio, dando continuidade aos objetivos atingidos com a intervenção realizada.

Sugere-se que sejam criados grupos de gestantes e puérperas, com a participação da equipe multiprofissional, bem como acompanhamento psicológico e nutricional, buscando o apoio mútuo para lidar com as alterações emocionais, sociais e prevenir possíveis intercorrências. Para isso, devem ser elaboradas atividades com temas construídos a partir das percepções do público-alvo ao longo das consultas e deve ser realizada a capacitação da equipe multiprofissional através do planejamento das atividades e ações. Deve-se realizar também a busca ativa de gestantes e puérperas, que não estejam comparecendo à UBS, durante as visitas domiciliares com o apoio do ACS, convocando-as para participação das atividades e continuidade ao acompanhamento.

Ademais, acreditamos que o aprendizado é construído e sempre podemos aprender com o outro e, dessa forma, legitimamos a importância de atividades que promovam a participação e a transformação de mais mulheres como essas, haja vista que a promoção da saúde é um fator fundamental para fomento de intervenções que visem melhorar a saúde e a qualidade de vida do indivíduo. 

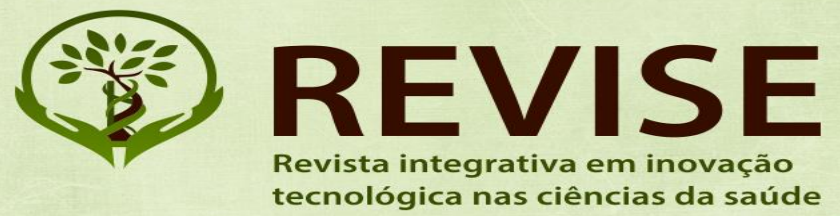

ISSN: $2179-6572$ tecnológica nas ciências da saúde
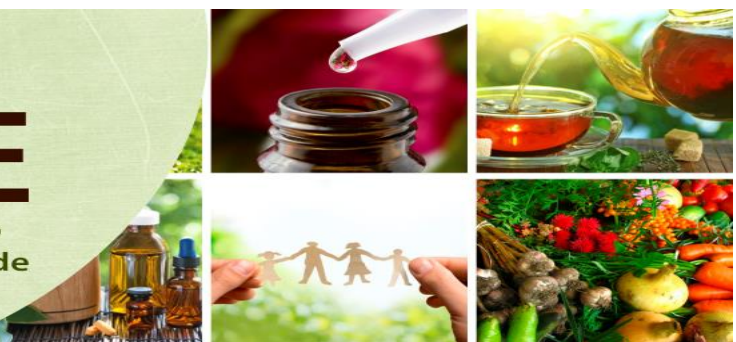

\section{REFERÊNCIAS}

ANDRADE, Raquel Dully, et. al. Fatores relacionados à saúde da mulher no puerpério e repercussões na saúde da criança. Escola Anna Nery, v. 19, n. 1, 2015, (181-186). Disponível em: https://doi.org/10.5935/1414-8145.20150025. Acesso em: 09/04/2020.

ANDRADE, Ana Carolina Vieira, et. al. Planejamento das ações educativas pela equipe multiprofissional da Estratégia Saúde da Família. O Mundo da Saúde, v. 37, n. 4, 2013, (439-449). Disponível em:

http://bvsms.saude.gov.br/bvs/artigos/mundo_saude/planejamento_acoes_educativas_equipe_multi profissional.pdf. Acesso em: 09/04/2020.

ANDRADE, Érica da Conceição; SILVA, Leila Rangel. Planejamento familiar: uma questão de escolha. Revista Eletrônica de Enfermagem, v. 11, 2009, (85-93). Disponível em:

https://doi.org/10.5216/ree.v11.46874. Acesso em: 09/04/2020.

ARAÚJO, Natalúcia Matos, et. al. Corpo e sexualidade durante a gravidez. Revista da escola de enfermagem da USP, v. 46, n. 3, 2012, (552-558). Disponível em:

http://www.scielo.br/pdf/reeusp/v46n3/04.pdf. Acesso em: 09/04/2020.

BRASIL. Constituição (1996). Lei No 9.263, de 12 de janeiro de 1996. Planejamento Familiar. Ministério da Saúde, 1996. Disponível em: http://www.planalto.gov.br/ccivil_03/leis/19263.htm. Acesso em: 09/04/2020.

BRASIL. Ministério da Saúde. Secretaria de Atenção à Saúde. Atenção ao pré-natal de baixo risco. Brasília: Editora do Ministério da Saúde, 2012. Disponível em:

https://bvsms.saude.gov.br/bvs/saudelegis/gm/2013/prt1020_29_05_2013.html. Acesso em: 09/04/2020.

BRASIL. Ministério da Saúde. Secretaria de Gestão Estratégica e Participativa. II Caderno de educação popular em saúde. Brasília: Ministério da Saúde, 2014. Disponível em:

https://bvsms.saude.gov.br/bvs/publicacoes/2_caderno_educacao_popular_saude.pdf. Acesso em: 09/04/2020.

BRUM, John Wesley Mota. O Desafio frente às contingências da dimensão psicossocial: Cuidando da gestante HIV positivo. Dissertação (Mestrado) - Curso de Enfermagem, Universidade Federal do Rio de Janeiro, Rio de Janeiro, 2013. Disponível em: http://objdig.ufrj.br/51/dissert/817756.pdf. Acesso em: 09 abr. 2020.

CONSELHO NACIONAL DE SAÚDE. Resolução no 466/2012. Diário Oficial da União. Seção 1, Brasília, DF, ano 2013, n. 112, p. 59, 13 jun. 2013. Disponível em: http://conselho.saude.gov.br/ultimas_noticias/2013/06_jun_14_publicada_resolucao.html. Acesso em: 03/04/2020.

Atenção Primária à Saúde. Revista Revise, v. 4, n. 00 (2020): O Sistema Único de Saúde na Formação e na Prática Médica, p. 116-131. 


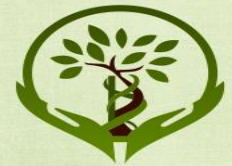

ISSN: $2179-6572$

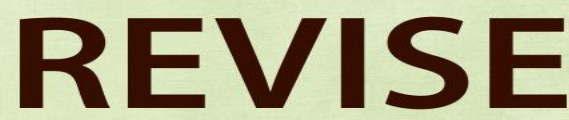

Revista integrativa em inovação tecnológica nas ciências da saúde

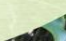

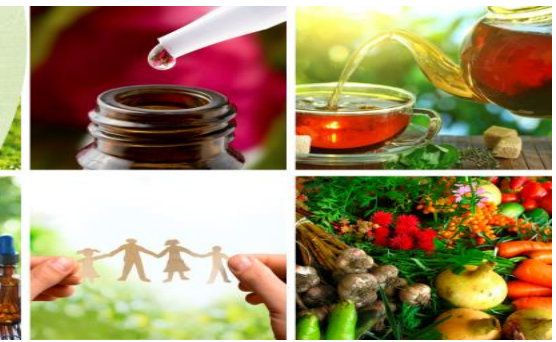

CONSELHO NACIONAL DE SAÚDE. Resolução n 510/2016. Diário Oficial da União. Seção 1, Brasília, DF, ano 2016, n. 98, p. 44, 24 maio 2016. Disponível em: http://www.in.gov.br/materia//asset_publisher/Kujrw0TZC2Mb/content/id/22917581. Acesso em: 03/04/2020

COSTA, Edina Silva, et. al. Alterações Fisiológicas na Percepção de Mulheres durante a Gestação. Revista da Rede de Enfermagem do Nordeste, v. 11, n. 2, abr./jun. 2010, (86-93). Disponível em: http://periodicos.ufc.br/rene/article/view/4531. Acesso em: 09/04/2020.

COUTINHO, Emília Carvalho, et. al. Gravidez e parto: o que muda no estilo de vida das mulheres que se tornam mães?. Revista da Escola de Enfermagem da Usp, v. 48, 2014, (17-24). Disponível em: http://www.scielo.br/pdf/reeusp/v48nspe2/pt_0080-6234-reeusp-48-nspe2-00017.pdf. Acesso em: 09/04/2020.

FREIRE, Paulo Reglus Neves. Educação como prática da liberdade. Rio de Janeiro: Paz e Terra, 2011.

INSTITUTO BRASILEIRO DE GEOGRAFIA E ESTATÍSTICA. Cidades e Estados. Munícipio de Itabuna (Bahia), 2018. Disponível em: https://www.ibge.gov.br/cidades-e-estados/ba/itabuna.html. Acesso em: 04/04/2020.

JARDIM, Mara Julyete Arraes, et. al. Contribuições do enfermeiro no pré-natal para a conquista do empoderamento da gestante. Cuidado é Fundamental, v. 11, 2019, (432-440). Disponível em: http://www.seer.unirio.br/index.php/cuidadofundamental/article/download/6370/pdf_1. Acesso em: 09/04/2020.

MANN, Luana, et. al. Alterações biomecânicas durante o período gestacional: uma revisão. Revista de Educação Física, v. 16, n. 3, jul./set.2010, (730-741). Disponível em:

http://www.scielo.br/scielo.php?pid=S1980-65742010000300022\&script=sci_abstract\&tlng=pt. Acesso em: 09/04/2020.

MOTTOLA, Michelle, et. al. 2019 Canadian guideline for physical activity throughout pregnancy. British Journal of Sports Medicine, v. 52, 2018, (1339-1346). Disponível em: https://bjsm.bmj.com/content/52/21/1339. Acesso em: 09/04/2020.

PICCININI, César Augusto, et. al. Gestação e constituição da maternidade. Psicologia em Estudo, v. 13, n. 1, jan./mar. 2008, (63-72). Disponível em:

http://www.scielo.br/pdf/pe/v13n1/v13n1a07.pdf. Acesso em: 09/04/2020.

PIO, Danielle Abdel Massih; OLIVEIRA, Mônica Martins de. Educação em saúde para atenção à gestante: paralelo de experiências entre Brasil e Portugal. Saude soc., v. 23, n. 1, jan./mar. 2014, (313-324). Disponível em: http://www.scielo.br/scielo.php?script=sci_arttext\&pid=S010412902014000100313\&lng=en\&nrm=iso. Acesso em: 09/04/2020. 


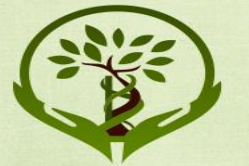

ISSN: $2179-6572$

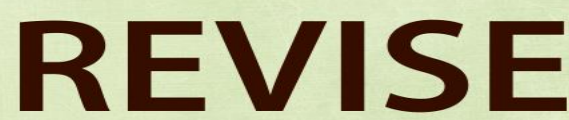

Revista integrativa em inovação tecnológica nas ciências da saúde
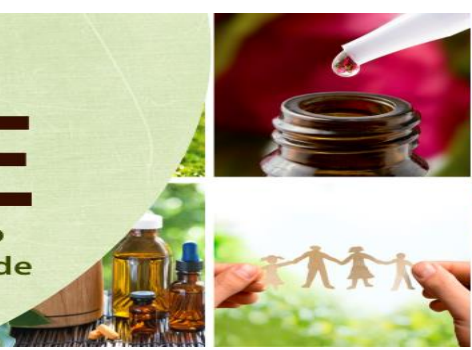

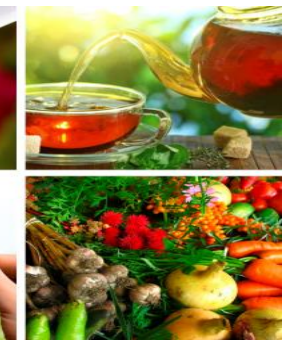

PRADO, Daniela Siqueira, et. al. Impacto da gestação na função sexual feminina. Revista Brasileira de Ginecologia e Obstetrícia, v. 35, n. 5, 2013, (205-209). Disponível em: http://www.scielo.br/scielo.php?pid=S0100-72032013000500003\&script=sci_abstract\&tlng=pt. Acesso em: 09/04/2020.

QUEROZ, Andréia Alcântara de. Conhecendo as alterações da gestação para um melhor cuidar do pré-natal. Doutorado em Atenção Básica em Saúde da Família. Universidade Federal de Minas Gerais, Brumadinho, 2012. Disponível em: https://www.nescon.medicina.ufmg.br/biblioteca/imagem/3819.pdf. Acesso em: 09/04/2020.

SAMPAIO, Juliana, et. al. Limites e potencialidades das rodas de conversa no cuidado em saúde: uma experiência com jovens no sertão pernambucano. Interface, v. 18, 2014, (12991311). Disponível em: https://doi.org/10.1590/1807-57622013.0264. Acesso em: 09/04/2020.

SILVA, Luzenilda Sabina, et. al. Análise das Mudanças Fisiológicas Durante a Gestação: desvendando mitos. Revista Faculdade Montes Belos, v. 8, n. 1, 2015, (1-16). Disponível em: http://revista.fmb.edu.br/index.php/fmb/article/view/11/8. Acesso em: 09/04/2020.

SILVEIRA, Carla Sofia Pascoal; FERREIRA, Manoela Maria Conceição. Autoconceito da Grávida: fatores associados. Millenium, n. 40, jun. 2011, (53-67). Disponível em: https://revistas.rcaap.pt/millenium/article/view/8219. Acesso em: 09/04/2020.

SOUZA, Viviane Barbosa, et. al. Ações educativas durante a assistência pré-natal: percepção de gestantes atendidas na rede básica de Maringá-PR. Revista Eletrônica de Enfermagem, v. 13, abr./jun. 2011, (199-210). Disponível em: https://doi.org/10.5216/ree.v13i2.10162. Acesso em: 09/04/2020.

SOUZA, Maria Luciana Martins. Rodas de conversas em saúde: uma estratégia de metodologia participativa no acompanhamento pré-natal de gestantes usuárias de um serviço de saúde. Especialização em Linhas de Cuidado em Enfermagem. Universidade Federal de Santa Catarina, Florianópolis, 2014. Disponível em:

https://repositorio.ufsc.br/bitstream/handle/123456789/172974/Maria\%20Luciana\%20Martins\%20d e\%20Souza\%20-\%20MATERNO\%20-\%20TCC.pdf?sequence=1\&isAllowed=y. Acesso em: $09 / 04 / 2020$.

TOSTES, Natalia Almeida; SEIDL, Eliane Maria Fleury. Expectativas de gestantes sobre o parto e suas percepções acerca da preparação para o parto. Trends in Psychology/Temas em Psicologia, v. 24, n. 2, 2016, (681-693). Disponível em: http://pepsic.bvsalud.org/pdf/tp/v24n2/v24n2a15.pdf. Acesso em: 09/04/2020.

VELHO, Manuela Beatriz, et. al. Parto normal e cesárea: representações sociais de mulheres que os vivenciaram. Revista Brasileira de Enfermagem, v. 67, n. 2, mar./abr. 2014, (282-289). Disponível em: https://doi.org/10.5935/0034-7167.20140038. Acesso em: 09/04/2020. 


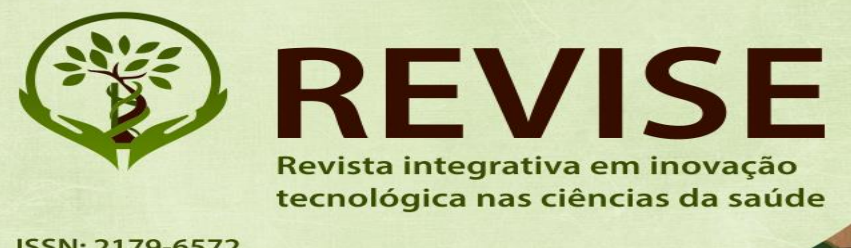

ISSN: $2179-6572$

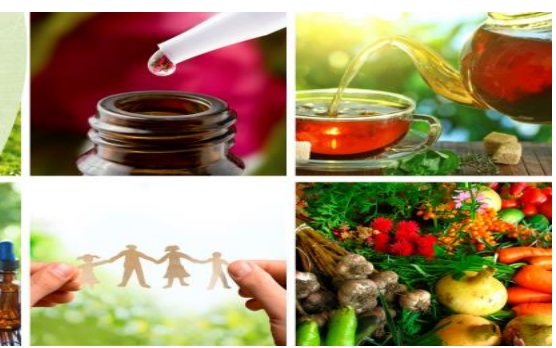

VIEIRA, Larissa da Silva, et. al. Gestação, Parto e Puerpério na Perspectiva das Gestantes de uma Unidade Básica de Saúde. 14 páginas. Portfólio de Atividades do Componente Curricular Propedêutica Clínica dos Problemas de Saúde da Gestação, Parto e Puerpério, Bacharelado Interdisciplinar em Saúde, Universidade Federal do Sul da Bahia, 2019. 